\title{
An Unusual Cause of Secondary ITP in a 34-Year-Old Hispanic Male
}

\author{
Ayrton Bangolo $\mathbb{D}^{1},{ }^{1}$ Mohamed Ahmed, ${ }^{1}$ Ali Atoot, ${ }^{2}$ Ashraf Mahmoud, ${ }^{3}$ \\ Chibuzo Agbakwuru-Onyike, ${ }^{1}$ Maria Karina Larni Y. Palad, ${ }^{4}$ Michael Sciarra, ${ }^{5}$ \\ and Adam Atoot ${ }^{1}$ \\ ${ }^{1}$ Department of Internal Medicine, Hackensack Meridian Health Palisades Medical Center, North Bergen, NJ, USA \\ ${ }^{2}$ Department of Anesthesia, Hackensack University Medical Center, Hackensack, NJ, USA \\ ${ }^{3}$ Department of Family Medicine, Hackensack Meridian Health Palisades Medical Center, North Bergen, NJ, USA \\ ${ }^{4}$ University of the East Ramon Magsaysay, Quezon, Philippines \\ ${ }^{5}$ Department of Gastroenterology, Hackensack Meridian Health Palisades Medical Center, North Bergen, NJ, USA
}

Correspondence should be addressed to Ayrton Bangolo; ayrtonbangolo@yahoo.com

Received 17 September 2021; Accepted 10 December 2021; Published 23 December 2021

Academic Editor: Tomas Jose Gonzalez-Lopez

Copyright (c) 2021 Ayrton Bangolo et al. This is an open access article distributed under the Creative Commons Attribution License, which permits unrestricted use, distribution, and reproduction in any medium, provided the original work is properly cited.

\begin{abstract}
Secondary immune thrombocytopenic purpura (ITP) associated with Helicobacter pylori (H. pylori) infection has been described in the literature. It appears to have a geographic distribution; mostly encountered in countries with a higher prevalence for H. pylori such as Italy or Japan. H. pylori eradication has been recommended in the management of ITP with studies showing improvement in the platelet count in some patients. Substantial platelet count increases in patients with severe thrombocytopenia (platelet counts $<30 \times 10^{3}$ microliter), however, are uncommon with $H$. pylori treatment alone. Here, we present a 34-year-old Hispanic male with worsening chronic thrombocytopenia that resolved following eradication of his $H$. pylori infection. Herein, we highlight a rare and reversible cause of secondary ITP. With this case report, we hope to encourage physicians to include $H$. pylori testing in the evaluation of thrombocytopenia.
\end{abstract}

\section{Introduction}

Immune thrombocytopenic purpura (ITP) is an acquired disorder in which there is immune-mediated destruction of platelets with possible inhibition of platelet release from the megakaryocytes [1]. In adults, it is a more chronic disease; however, some will experience spontaneous remission which will usually occur within months of the initial diagnosis. ITP is considered secondary if it is associated with an underlying disorder [1]. Several studies have recently shown improvement in platelet counts after eradicating Helicobacter pylori (H. pylori) infection which suggests an association between the two conditions [2]. It appears that response rates are higher in countries where H. pylori infection is endemic and in patients with milder degrees of thrombocytopenia [3, 4]. Herein, we present a 34-year-old male with
ITP and worsening platelet levels who was found to have H. pylori infection. Following treatment of his infection, the patient had significant improvement and normalization of his platelet count. With this case report, we aim to encourage clinicians to include $H$. pylori in the differential diagnosis of ITP.

\section{Case Presentation}

This is a 34-year-old Hispanic male with no significant past medical history who presented to the clinic for evaluation of a gnawing, moderate, intermittent epigastric pain that started 3-4 months prior to the visit. The pain improves with meals and is associated with reflux and early satiety. Notably, the patient was told he had "low platelets" on his annual physical the year prior to our encounter. He denied 
TABLE 1: Laboratory values.

\begin{tabular}{|c|c|}
\hline & Laboratory values \\
\hline White blood cells & $5.5 \times 10^{*} 3\left(3.4-10.8 \times 10^{*} 3\right)$ microliter $(\mathrm{uL})$ \\
\hline Red blood cells & $5.0 \times 10^{*} 6\left(4.14-5.80 \times 10^{*} 6\right) \mathrm{uL}$ \\
\hline Platelet count on admission & $50 \times 10^{*} 3\left(150-450 \times 10^{*} 3\right) \mathrm{uL}$ \\
\hline Platelet count a year ago & $91 \times 10^{*} 3\left(150-450 \times 10^{*} 3\right) \mathrm{uL}$ \\
\hline Platelet count 1 week into therapy & $105 \times 10^{*} 3\left(150-450 \times 10^{*} 3\right) \mathrm{uL}$ \\
\hline Platelet count 5 weeks after triple therapy & $178 \times 10^{*} 3\left(150-450 \times 10^{*} 3\right) \mathrm{uL}$ \\
\hline Erythrocyte sedimentation rate & $3(0-15)$ millimeter per hour $(\mathrm{mm} / \mathrm{hr})$ \\
\hline C-reactive protein & $<1(0-10)$ milligram per liter $(\mathrm{mg} / \mathrm{L})$ \\
\hline Hepatitis B surface antigen & Negative \\
\hline Hepatitis B surface antibody, quantitative & $\begin{array}{c}>10,000 \text { (immunity }>9.9) \text { milli-international units per milliliter } \\
(\mathrm{mIU} / \mathrm{mL})\end{array}$ \\
\hline Aspartate aminotransferase & $19(0-40)$ international units per liter (IU/L) \\
\hline Alanine transaminase & $25(0-44) \mathrm{IU} / \mathrm{L}$ \\
\hline Human immunodeficiency virus antigen/antibody 4 th generation & Nonreactive \\
\hline Hepatitis $\mathrm{C}$ antibody & $<0.1(0.0-0.9)$ signal-to-cutoff ratio (s/co ro) \\
\hline Antinuclear antibody & Negative \\
\hline Serotonin release assay & Negative \\
\hline
\end{tabular}

any diarrhea, nausea, vomiting, spontaneous bruising, epistaxis, or gingival bleeding. He also denied any intravenous drug use or any dangerous sexual behaviors. He has never been hospitalized and did not undergo any surgical procedures in the past. The family history was noncontributory.

Physical examination was significant for epigastric tenderness on palpation but was otherwise unremarkable. Laboratory results revealed a positive urea breath test as well as worsening thrombocytopenia with a platelet count of $50 \times 10^{3}$ microliter $(\mathrm{uL})\left(150-450 \times 10^{3}\right)$ from a platelet count of $91 \times 10^{3} \mathrm{uL}$ the year prior as shown in Table 1 . The remainder of the laboratory evaluation, including a complete blood count, lipid profile, and urinalysis was unremarkable. The abdominal ultrasound was unremarkable except for a trace effusion between the liver and diaphragm. Further evaluation with a noncontrast computed tomography (CT) of the abdomen/pelvis showed no abdominal pathology.

The patient was started on a 10-day regimen triple therapy (amoxicillin, clarithromycin, and lansoprazole) for eradication of Helicobacter pylori (H. pylori). One week after initiation of therapy, repeat laboratory results revealed significantly improved platelet levels, $105 \times 10^{3} \mathrm{uL}$. Additional workup for thrombocytopenia was ordered including a hepatitis panel, human immunodeficiency virus testing, heparin-induced thrombocytopenia antibodies, and a serotonin release assay which were all within normal limits.

The patient was reevaluated 5 weeks after completion of the triple therapy. He reported complete resolution of his abdominal pain. The urea breath test was negative as well as the stool antigen for $H$. pylori. The laboratory results revealed continuous improvement and normalization of the platelet levels, $178 \times 10^{3} \mathrm{uL}$.

\section{Discussion}

Immune thrombocytopenia (ITP, also called idiopathic thrombocytopenic purpura or immune thrombocytopenic purpura) is an acquired thrombocytopenia caused by autoantibodies against platelet antigens. It is one of the more common causes of thrombocytopenia in otherwise asymptomatic adults [1]. ITP is termed secondary if it is associated with an underlying disorder; autoimmune disorders, particularly systemic lupus erythematosus (SLE), and infections, such as human immunodeficiency virus (HIV) infections and hepatitis $\mathrm{C}$, are also common causes. The association of ITP with Helicobacter pylori (H. pylori) infection is unclear [1]. Some studies show that eradication of $H$. pylori with antibiotics result in marked platelet count increases in patients with ITP [3]. Patients with acute $H$. pylori are often asymptomatic or develop mild self-limited dyspeptic symptoms and may not seek medical attention and thus are not immediately investigated [5]. Our patient had a one-year history of acquired unexplained thrombocytopenia and developed dyspeptic symptoms overtime. He was not initially worked up for $H$. pylori a year prior to our encounter, given the lack of dyspeptic symptoms at the time. He did not endorse any signs or symptoms related to thrombocytopenia. The patient also denied any personal or family history of bleeding disorders or autoimmune conditions.

ITP is a diagnosis of exclusion which is made in patients with isolated thrombocytopenia. The diagnosis of ITP requires that other potential causes of thrombocytopenia, such as recent infections, medications, rheumatologic disorders, or liver disease, be excluded [6]. Several guidelines recommend obtaining a peripheral blood smear to confirm that thrombocytopenia is not artifactual due to platelet clumping and HIV and hepatitis C testing [6-8]. Testing for $H$. pylori is appropriate in patients with gastrointestinal symptoms suggestive of infection because of a reported association between ITP and $H$. pylori infection. Routine screening for $H$. pylori may be reasonable in asymptomatic individuals as well [4]. Antiplatelet antibody testing has low sensitivity and is not recommended [9]. Our patient was not taking any medications including any herbal supplementation to explain his findings. He had no 
history of rheumatologic disorders, and the liver function test was unremarkable. A year prior to our encounter, the patient had unexplained thrombocytopenia but did not have any gastrointestinal symptoms. It was his dyspepsia and early satiety a year later that prompted evaluation for $H$. pylori infection. However, even despite the lack of dyspeptic symptoms, the patient should have been evaluated for $H$. pylori as part of the workup of unexplained thrombocytopenia.

The treatment of ITP uses drugs that decrease reticuloendothelial uptake of the antibody-bound platelet, decrease antibody production, and/or increase platelet production. Patients with platelet counts $>30 \times 10^{3} \mu \mathrm{L}$ appear not to have increased mortality related to the thrombocytopenia and usually do not require treatment with intravenous immune globulin (IVIG) or corticosteroids [1]. IVIG or corticosteroids are usually reserved for bleeding, elective surgery, or severe thrombocytopenia $\left(<30 \times 10^{3}\right.$ microliter $)$ without bleeding [10]. A systematic review of the English literature concluded that, in secondary ITP, there is improvement of thrombocytopenia with $H$. pylori eradication [4]. Our patient had a platelet level above $30 \times 10^{3} \mathrm{uL}$, was not bleeding, and was not scheduled for any surgeries. Thus, he was not treated with corticosteroids or IVIG. He was found to have a positive urea breath test and was initiated on triple therapy for $H$. pylori eradication. Following H. pylori eradication, the platelet levels normalized, an outcome that is consistent with findings in the literature.

\section{Conclusion}

Immune thrombocytopenic purpura (ITP) is an acquired disorder that can be associated with Helicobacter pylori (H. pylori) as previously reported in the literature. H. pylori eradication has been associated with improvement and normalization of the platelet levels, especially in patients with nonsevere thrombocytopenia. With this case report, we hope to contribute to the growing literature of $H$. pylori associated with ITP. This case report has the objective to encourage clinicians to include $H$. pylori in the workup of unexplained thrombocytopenia, even without gastrointestinal symptoms.

\section{Data Availability}

All data generated or analyzed during this study are available from the corresponding author upon request.

\section{Ethical Approval}

This study protocol was reviewed and the need for approval was waived by the ethics committee at Palisades Medical Center Hackensack Meridian Health.

\section{Consent}

Written informed consent was obtained from the patient for publication of this case report and any accompanying images.

\section{Disclosure}

Adam Atoot and Michael Sciarra are the article's guarantors.

\section{Conflicts of Interest}

The authors declare that they have no conflicts of interest.

\section{Authors' Contributions}

Ayrton Bangolo and Mohamed Ahmed searched the literature and wrote and revised the manuscript. Ali Atoot, Ashraf Mahmoud, Chibuzo Agbakwuru-Onyike, and Maria Karina Larni Y. Palad revised and edited the manuscript. Adam Atoot and Michael Sciarra revised and approved the final version. All authors certify that they contributed sufficiently to the intellectual content and data analysis. Each author has reviewed the final version of the manuscript and approved it for publication.

\section{References}

[1] B. A. Konkle, "Disorders of platelets and vessel wall," in Harrison's Principles of Internal Medicine, J. L. Jameson, A. S. Fauci, D. L. Kasper, S. L. Hauser, D. L. Longo, and J. Loscalzo, Eds., McGraw-Hill Education, New York, NY, USA, 2018.

[2] N. Rostami, M. Keshtkar-Jahromi, M. Rahnavardi, M. Keshtkar-Jahromi, and F. Soghra Esfahani, "Effect of eradication of Helicobacter pylori on platelet recovery in patients with chronic idiopathic thrombocytopenic purpura: a controlled trial," American Journal of Hematology, vol. 83, no. 5, pp. 376-381, 2008.

[3] K. Kaushansky, "Thrombocytopenia," in Williams Hematology, K. Kaushansky, J. T. Prchal, L. J. Burns, M. A. Lichtman, M. Levi, and D. C. Linch, Eds., McGraw-Hill Education, New York, NY, USA, 2021.

[4] R. Stasi, A. Sarpatwari, J. B. Segal et al., "Effects of eradication of Helicobacter pylori infection in patients with immune thrombocytopenic purpura: a systematic review," Blood, vol. 113, no. 6, pp. 1231-1240, 2009.

[5] G. A. Rocha, D. M. Queiroz, E. N. Mendes, A. J. Barbosa, G. F. Lima Júnior, and C. A. Oliveira, "Helicobacter pylori acute gastritis: histological, endoscopical, clinical, and therapeutic features," American Journal of Gastroenterology, vol. 86, no. 11, pp. 1592-1595, 1991.

[6] British Committee for Standards in Haematology General Haematology Task Force, "Guidelines for the investigation and management of idiopathic thrombocytopenic purpura in adults, children and in pregnancy," British Journal of Haematology, vol. 120, no. 4, pp. 574-596, 2003.

[7] D. B. Cines and V. S. Blanchette, "Immune thrombocytopenic purpura," New England Journal of Medicine, vol. 346, no. 13, pp. 995-1008, 2002.

[8] F. Rodeghiero, R. Stasi, T. Gernsheimer et al., "Standardization of terminology, definitions and outcome criteria in immune thrombocytopenic purpura of adults and children: report from an international working group," Blood, vol. 113, no. 11, pp. 2386-2393, 2009.

[9] J. George, S. Woolf, G. Raskob et al., "Idiopathic thrombocytopenic purpura: a practice guideline developed by explicit 
methods for the American Society of Hematology," Blood, vol. 88, no. 1, pp. 3-40, 1996.

[10] B. Godeau, S. Chevret, B. Varet et al., "Intravenous immunoglobulin or high-dose methylprednisolone, with or without oral prednisone, for adults with untreated severe autoimmune thrombocytopenic purpura: a randomised, multicentre trial," The Lancet, vol. 359, no. 9300, pp. 23-29, 2002. 